\title{
FRAMEWORK AND METHOD FOR MODEL BASED DIAGNOSIS WITH APPLICATION TO AN AUTOMOTIVE ENGINE
}

\author{
Mattias Nyberg \\ Department of Electrical Engineering, Linköping University \\ S-581 83, Linköping, Sweden \\ Email: matny@isy.liu.se, Fax: +46-13-282035
}

\begin{abstract}
A general framework and a method for model based diagnosis are suggested. In the framework, faults and modeling of faults are formally described. Then the method is used to construct a diagnosis system based on a structure of hypothesis tests. Using these principles, it is possible to diagnose a large variety of different types of faults. The framework and the method are applied to the design of a diagnosis system for an automotive engine. This application clearly shows the strength of these principles because several different types of sensor faults and leakage need to be diagnosed. The resulting diagnosis system is demonstrated using experiments on a real automotive engine.
\end{abstract}

\section{Introduction}

Model based fault diagnosis has gained increased interest during the last 10 years. Reasons are the availability of cheap and powerful microprocessors together with increased demand on diagnosis performance in many areas, e.g. automotive engines. Many studies have been devoted to theory, e.g. see the reviews [1][2], but few results have shown the practical use in real applications.

One problem with the previously presented diagnosis methods is that they are each specialized to diagnose a certain type of faults. On the contrary, real application contain rarely only one type of faults. Instead, the common situation is that there is a need to diagnose many different types of faults.

As an attempt towards solving the above described problems, we here suggest a new framework in which all kinds of diagnosis problems can be described. Also presented is a new method structured hypothesis tests. Both the framework and the method are quite general and therefore probably more useful in real applications than many other principles. In addition, much more model knowledge can be included in the design of the diagnosis system, which implies that there is a high potential to obtain better diagnosis performance.

The framework is described in Section 2 and the principle of structured hypothesis tests is described in Section 3. These are then applied to the diagnosis of the air-intake system of an automotive engine in Section 4 and 5 . It is seen that a large variety of different faults can be diagnosed. In Section 6 the diagnosis system is then experimentally validated.

\section{Fault Modeling and Fault Modes}

This section presents the new framework for describing diagnosis problems. Within this framework, we can formally describe faults and the modeling of faults. Only a short description is given here, but a thorough discussion can be found in [3].

A system can usually be separated into a number of components. For each of these components a number of faults can occur. Each of these faults can be classified into different fault modes. For example, one component may be an angle sensor and all bias faults, regardless of the size of the bias, belongs to the same fault mode. We use the convention that one of the fault modes is the no fault case. From this perspective, the characteristic property of a component is that only one fault mode can be present at a time. Consider for example a valve with fault modes "no fault", "stuck open", and "stuck closed". Obviously no two of these fault modes can be present at the same time. It is also possible to talk about fault modes for the whole system. To avoid confusion, we will often use the terms component fault-mode and system fault-mode.

Let $F_{j}^{i}$ be the $j$ :th component fault-mode of the $i$ :th component. We will reserve the fault-mode $F_{0}^{i}$ to be the "no fault" case of the $i$ :th component. The fault-mode $F_{0}^{i}$ will also be denoted $N F^{i}$. Further let $p$ be the number of components and $n_{i}$ the number of different component faultmodes for the $i$ :th component. All component fault-modes can then be collected in a table:

\begin{tabular}{l|l}
$\begin{array}{l}\text { component } \\
\text { number } i\end{array}$ & $\begin{array}{l}\text { component } \\
\text { fault-modes }\end{array}$ \\
\hline 1 & $F_{0}^{1} \equiv N F^{1}, F_{1}^{1}, \ldots F_{n_{1}}^{1}$ \\
2 & $F_{0}^{2} \equiv N F^{2}, F_{1}^{2}, \ldots F_{n_{2}}^{2}$ \\
$\vdots$ & $\vdots$ \\
$\mathrm{p}$ & $F_{0}^{p} \equiv N F^{p}, F_{1}^{p}, \ldots F_{n_{p}}^{p}$
\end{tabular}

A system fault-mode can then be composed by a vector of component fault-modes. Thus the length of this vector is $p$. The total number of possible system fault-modes are $\prod_{i} n_{i}$. We will use bold-face letters to denote system fault-modes. Some examples of system fault-modes are

$$
\begin{aligned}
\mathbf{N F} & =\left[N F^{1}, N F^{2}, \ldots N F^{p}\right] \\
\mathbf{F}_{\mathbf{1}}^{\mathbf{1}} & =\left[F_{1}^{1}, N F^{2}, \ldots N F^{p}\right] \\
\mathbf{F}_{\mathbf{1}}^{\mathbf{2}} & =\left[N F^{1}, F_{1}^{2}, N F^{3}, \ldots N F^{p}\right] \\
\mathbf{F}_{\mathbf{2}}^{\mathbf{1}} \& \mathbf{F}_{\mathbf{1}}^{\mathbf{2}} & =\left[F_{2}^{1}, F_{1}^{2}, N F^{3}, \ldots N F^{p}\right]
\end{aligned}
$$


The first of these examples is the no-fault case of the whole system. For the other examples, we have used the convention that components, that have none of its component fault-modes included in the notation for the system faultmode, are assumed to have component fault-mode $N F^{i}$.

The system fault-modes in which only one of the component fault-modes is not $N F^{i}$ are said to be single faultmodes. For example, $\mathbf{F}_{\mathbf{1}}^{\mathbf{1}}$ and $\mathbf{F}_{\mathbf{1}}^{\mathbf{2}}$ in the example above, are single fault-modes. Usually also the no-fault system faultmode, i.e. NF, is said to be a single fault-mode. Often, only single fault-modes are considered. This corresponds to an assumption that only one fault can be present at the same time. Then the number of possible system fault-modes becomes $1+\sum_{i}\left(n_{i}-1\right)$.

\subsection{Fault States}

Each component $i$ has a parameter $\theta_{i}$ which determines the exact fault, or fault state, (which can be no fault) of the component. The parameter space of $\theta_{i}$ is denoted $\mathcal{D}^{i}$. Then each component fault-mode is associated with a subspace $\mathcal{D}_{F_{j}^{i}}^{i}$ of $\mathcal{D}^{i}$. That is, if fault mode $F_{j}^{i}$ is present in component $i$, then $\theta_{i} \in \mathcal{D}_{F_{j}^{i}}^{i}$.

The component parameters $\theta_{i}$ are collected in a vector $\theta=\left[\theta_{1}, \ldots \theta_{p}\right]$ that represents the fault state of the whole system. The parameter space of $\theta$ is $\Theta=\mathcal{D}^{1} \times \cdots \times \mathcal{D}^{p}$. Each system fault-mode $\gamma$ is associated with a subspace $\Theta_{\gamma}$ of $\Theta$. This means that $\Theta=\bigcup_{\gamma} \Theta_{\gamma}$. For the single fault-modes in the examples (1) we have

$$
\begin{array}{ll}
\mathbf{N F} & \theta \in \Theta_{\mathbf{N F}}=\left\{\theta \in \Theta \mid \bigwedge_{i} \theta_{i} \in \mathcal{D}_{N F^{i}}^{i}\right\} \\
\mathbf{F}_{\mathbf{1}}^{\mathbf{1}} & \theta \in \Theta_{\mathbf{F}_{1}^{1}}=\left\{\theta \in \Theta \mid \theta_{1} \in \mathcal{D}_{F_{1}^{1}}^{1} \bigwedge_{i \neq 1} \theta_{i} \in \mathcal{D}_{N F^{i}}^{i}\right\} \\
\mathbf{F}_{\mathbf{1}}^{\mathbf{2}} & \theta \in \Theta_{\mathbf{F}_{\mathbf{1}}^{2}}=\left\{\theta \in \Theta \mid \theta_{2} \in \mathcal{D}_{F_{1}^{2}}^{2} \bigwedge_{i \neq 2} \theta_{i} \in \mathcal{D}_{N F^{i}}^{i}\right\}
\end{array}
$$

Note that in this framework, where faults are modeled as parameters, it is possible to also describe faults that are traditionally modeled as signals. In this case a signal is seen as a parameter vector.

\subsection{Models}

The whole system model will be denoted $\mathcal{M}(\theta)$. The model $\mathcal{M}(\theta)$ with a fixed value of $\theta$ then exactly specifies the system when a specific fault (or no fault) is present. We will also use the notation $\mathcal{M}_{\gamma}(\theta)$ denoting a model of the system when fault mode $\gamma$ is present, i.e. $\mathcal{M}_{\gamma}(\theta)=\left.\mathcal{M}(\theta)\right|_{\theta \in \Theta_{\gamma}}$.

\section{Model Based Diagnosis using Structured Hypothesis Tests}

This section discusses how diagnosis system can be constructed using a structure of hypothesis tests. The method can be seen as a generalization of the method structured residuals [1]. This link and also other aspects of the method are thoroughly discussed in [3].

A key feature of the method is that the model of the system can be fully utilized in a systematic way. Further, the method is intuitive and very similar to the reasoning involved when humans are doing diagnosis. Using hypothesis testing the way it is done here, is quite general and several other principles for diagnosis can be seen as special cases, e.g. parameter estimation, observer schemes, and statistical approaches.

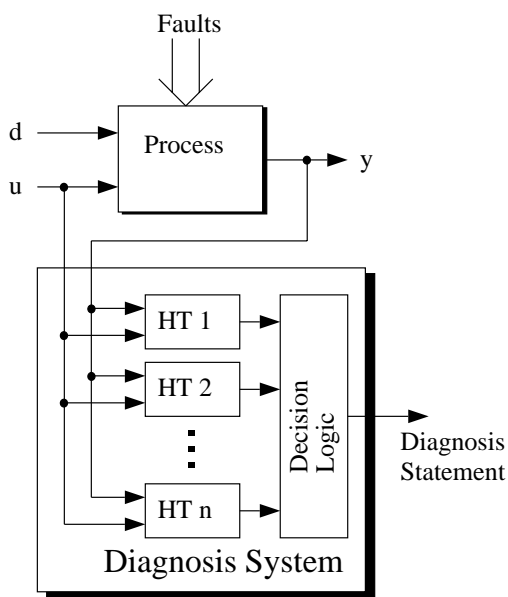

Figure 1: The diagnosis system.

The diagnosis system has a structure according to Figure 1. The inputs to the diagnosis system is the process input $u$ and the process output $y$. The signal $d$ represents inputs that are unknown to the diagnosis system, e.g. disturbances. The output of the diagnosis system is the $d i$ agnosis statement, which contains information about which fault modes that can explain the behavior of the process. The diagnosis system consists of a set of hypothesis tests, HT 1 to $H T$ n, and the decision logic.

The null hypothesis for the $i$ :th hypothesis test, i.e. $H_{i}^{0}$, is that the system fault-mode, present in the process, belongs to a specific set $M_{i}$ of system fault-modes. The alternative hypothesis $H_{i}^{1}$ is that the present fault mode does not belong to $M_{i}$. This means that if hypothesis $H_{i}^{0}$ is rejected, the present fault mode can not belong to $M_{i}$. In this way, each separate hypothesis test contributes with a piece of informations about which fault modes that can be present. The purpose of the decision logic is then to combine this information to form the diagnosis statement.

For example, let $\mathbf{F}_{\mathbf{p}}$ denote the present system fault-mode and assume that the diagnosis system contains the following set of three hypothesis tests:

$$
\begin{array}{ll}
H_{1}^{0}: \mathbf{F}_{\mathbf{p}} \in M_{1}=\left\{\mathbf{N F}, \mathbf{F}_{\mathbf{1}}\right\} & H_{1}^{1}: \mathbf{F}_{\mathbf{p}} \in M_{1}^{C}=\left\{\mathbf{F}_{\mathbf{2}}, \mathbf{F}_{\mathbf{3}}\right\} \\
H_{2}^{0}: \mathbf{F}_{\mathbf{p}} \in M_{2}=\left\{\mathbf{N F}, \mathbf{F}_{\mathbf{2}}\right\} & H_{2}^{1}: \mathbf{F}_{\mathbf{p}} \in M_{2}^{C}=\left\{\mathbf{F}_{\mathbf{1}}, \mathbf{F}_{\mathbf{3}}\right\} \\
H_{3}^{0}: \mathbf{F}_{\mathbf{p}} \in M_{3}=\left\{\mathbf{N F}, \mathbf{F}_{\mathbf{3}}\right\} & H_{3}^{1}: \mathbf{F}_{\mathbf{p}} \in M_{3}^{C}=\left\{\mathbf{F}_{\mathbf{1}}, \mathbf{F}_{\mathbf{2}}\right\}
\end{array}
$$

Then if only $H_{1}^{0}$ is rejected, we can draw the conclusion that $\mathbf{F}_{\mathbf{p}} \in M_{1}^{C}=\left\{\mathbf{F}_{\mathbf{2}}, \mathbf{F}_{\mathbf{3}}\right\}$, i.e. the present system fault-mode is either $\mathbf{F}_{\mathbf{2}}$ or $\mathbf{F}_{\mathbf{3}}$. If both $H_{1}^{0}$ and $H_{2}^{0}$ are rejected, we can draw the conclusion that $\mathbf{F}_{\mathbf{p}} \in M_{1}^{C} \cap M_{2}^{C}=\left\{\mathbf{F}_{\mathbf{2}}, \mathbf{F}_{\mathbf{3}}\right\} \cap$ $\left\{\mathbf{F}_{\mathbf{1}}, \mathbf{F}_{\mathbf{3}}\right\}=\left\{\mathbf{F}_{\mathbf{3}}\right\}$, i.e. the present system fault-mode is $\mathbf{F}_{\mathbf{3}}$.

From the above example, it is clear that the decision logic is a simple intersection operation. The diagnosis statement $S$ then becomes a set of fault modes that can be expressed as

$$
S=\bigcap_{\substack{i \\ H_{i}^{1} \text { accepted }}} M_{i}^{C}
$$

\subsection{Hypothesis Tests}

Much of the engineering work involved in constructing a diagnosis system is to use the model of the system, i.e. all 
$\mathcal{M}_{\gamma}(\theta)$, to construct the individual hypothesis tests. However, the first step is to decide the set of hypotheses to test. Here we will consider only single fault-modes and for this case, it is natural to use one hypothesis test for each system fault-mode. Thus the set of hypothesis tests can be indexed by $\gamma$, i.e. $H T_{\gamma}$, and becomes

$$
\begin{aligned}
& H_{\gamma}^{0}: \mathbf{F}_{\mathbf{p}} \in M_{\gamma} \\
& H_{\gamma}^{1}: \mathbf{F}_{\mathbf{p}} \in M_{\gamma}^{C} \\
& \gamma \in\left\{\mathbf{N F}, \mathbf{F}_{\mathbf{1}}^{\mathbf{1}}, \ldots \mathbf{F}_{\mathbf{n}_{\mathbf{1}}}^{\mathbf{1}}, \mathbf{F}_{\mathbf{0}}^{\mathbf{2}}, \ldots \mathbf{F}_{\mathbf{n}_{\mathbf{p}}}^{\mathbf{p}}\right\}
\end{aligned}
$$

It turns out that some system fault-modes are related to other system fault modes such that for some values of $\theta$ they are impossible to separate. This has implications on how the sets $M_{\gamma}$ can be chosen. For example for most fault modes, the limit when the fault size goes to zero is equal to the fault mode "no fault". This means that when fault mode NF, i.e. no fault, is present, most null hypothesis can not be rejected. The implication is that almost all sets $M_{\gamma}$ must include NF.

For each hypothesis test $H T_{\gamma}$, we need to find a test quantity and a rejection region. The sample data $\mathbf{x}$ for each hypothesis is

$$
\mathbf{x}=\left[\begin{array}{llll}
u\left(t_{0}\right) & u\left(t_{0}+1\right) & \ldots & u\left(t_{0}+N\right) \\
y\left(t_{0}\right) & y\left(t_{0}+1\right) & \ldots & y\left(t_{0}+N\right)
\end{array}\right]
$$

To simplify the notation, we have assumed that unit sampletime is used. The test quantity is a function $T_{\gamma}(\mathbf{x})$ from the sample data $\mathbf{x}$, to a scalar value which is to be thresholded by a threshold $J_{\gamma}$. Thus the rejection region is defined implicitly by the threshold $J_{\gamma}$ together with the test quantity $T_{\gamma}(\mathbf{x})$. The hypothesis test $\mathrm{HT}_{\gamma}$ is then defined as

$$
\begin{array}{ll}
T_{\gamma}(\mathbf{x})>J_{\gamma} & \text { reject } H_{\gamma}^{0}, \text { i.e. accept } H_{i}^{1} \\
T_{\gamma}(\mathbf{x})<J_{\gamma} & \text { do not reject } H_{\gamma}^{0}
\end{array}
$$

This means that we need to design a test quantity $T_{\gamma}(\mathbf{x})$ such that it is low if the data $\mathbf{x}$ match the hypothesis $H_{\gamma}^{0}$, i.e. a fault mode in $M_{\gamma}$ can explain the data. Also if the data come from a fault mode not in $M_{\gamma}, T_{\gamma}(\mathbf{x})$ should be large.

As test quantity, we will use the following function:

$$
T_{\gamma}(\mathbf{x})=\min _{\theta \in \Theta_{\gamma}} V_{\gamma}(\theta, \mathbf{x})
$$

where $V_{\gamma}(\theta, \mathbf{x})$ is a measure measuring the validity of the model $\mathcal{M}_{\gamma}(\theta)$ with respect to the data $\mathbf{x}$. In the special case when the fault mode $\gamma$ contains only one specific fault, i.e. the fault mode corresponds to one specific value of $\theta$, then $\Theta_{\gamma}$ contains only one element and the model can be written $\mathcal{M}_{\gamma}$. The test quantity for this case becomes

$$
T_{\gamma}(\mathbf{x})=V_{\gamma}(\mathbf{x})
$$

The details on how the test quantities are constructed depends on the actual case and only for some specific classes of systems, general design procedures have been proposed, e.g. linear systems.

\section{Application to an Automotive Engine}

This section and the next section describes how the diagnosis of the air-intake system of a real automotive engine can be described and solved using the framework from the two previous sections. The problem is to diagnose both sensor faults and leakage. In two previous works, [4] and [5], these two diagnosis problems were solved by using two different methods. However, each of these methods is dedicated to a certain type of faults and the two methods can not easily be combined into one single diagnosis system capable of diagnosing both leaks and sensor faults.

\subsection{Engine Description}

The engine considered is 4 cylinder, 2.3 liter, turbo-charged, spark-ignited SAAB production engine. Both during the model building and the validation, the engine was run according to the FTP-75 test-cycle.

A schematic picture of the air-intake system is shown in Figure 2. Ambient air enters the system and an air-mass flow sensor measures the air-mass flow rate $\dot{m}$. Next, the air passes the compressor side of the turbo-charger and then the intercooler. This results in a boost pressure $p_{b}$ that is higher than ambient pressure. Next, the air passes the throttle and this flow is denoted $\dot{m}_{t h}$. Finally the air leaves the manifold and enters the cylinder and this flow is denoted $\dot{m}_{c y l}$. Also shown in the figure are the two possible leaks: the boost leak somewhere between the air-mass flow sensor and the throttle, and the manifold leak somewhere in the manifold.

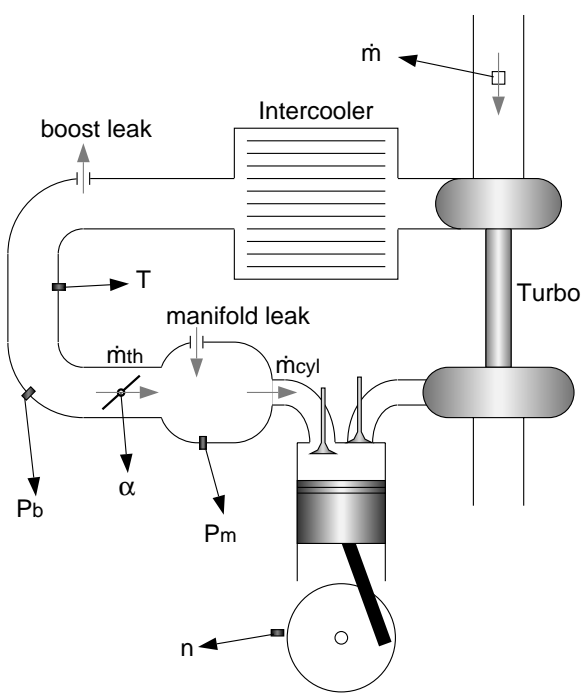

Figure 2: The turbo-charged engine. Air-mass flows that are discussed in the text are marked with gray arrows.

\subsection{Modeling}

In this work, the air-intake system is modeled with only static relations. This is sufficient because there is no need for extremely fast detection of leakage or sensor faults. The modeling work, including identification of parameters, was presented in [5]. Below we give a short description of the model.

The model for the fault-free system is described by the following equations

$$
\begin{aligned}
\dot{m} & =\dot{m}_{t h} \\
\dot{m}_{t h} & =\dot{m}_{c y l}
\end{aligned}
$$


where $\dot{m}_{t h}=f\left(p_{b}, \alpha, p_{m}\right)$ and $\dot{m}_{c y l}=g\left(p_{m}, n\right)$. The functions $f\left(p_{b}, \alpha, p_{m}\right)$ and $g\left(p_{m}, n\right)$ are both non-linear and static.

A manifold leak will mostly result in an air flow $\dot{m}_{\text {manLeak }}$ in the direction into the manifold. This air flow is described by $\dot{m}_{\text {manLeak }}=k_{m} h_{m}\left(p_{m}\right)$, where $k_{m}$ is the area of the leakage hole and $h_{m}\left(p_{m}\right)$ is a non-linear and static function.

The model for the whole air-intake system with manifold leak present is obtained by replacing Equation (7b) with

$$
\dot{m}_{t h}+\dot{m}_{\text {manLeak }}=\dot{m}_{c y l}
$$

The boost leakage model is similar and for more details about the model, see [5]. Both leakage models were validated in [5] and good agreement with real data was obtained.

\section{Construction of the Diagnosis System}

This section presents the design of a diagnosis system for the air-intake system. The objective is not to present a complete design but rather to give some examples that illustrates how the principles from Section 2 and 3 can be used in practice.

\begin{tabular}{|c|c|c|}
\hline $\begin{array}{l}\text { index } \\
i\end{array}$ & $\begin{array}{l}\text { component } \\
\text { name }\end{array}$ & $\begin{array}{l}\text { component } \\
\text { fault modes }\end{array}$ \\
\hline$B$ & boost pipe & $N F^{B}, B L$ \\
\hline$M$ & manifold & $N F^{M}, M L$ \\
\hline$B S$ & boost pressure sensor & $N F^{B S}, B B, B A F$ \\
\hline$M S$ & manifold pressure sensor & $N F^{M S}, M G, M C$ \\
\hline$T S$ & throttle sensor & $N F^{T S}, T L F$ \\
\hline$A S$ & air mass-flow sensor & $N F^{A S}, A L C$ \\
\hline
\end{tabular}

Table 1: The components and component fault-modes considered.

The different component fault-modes that will be considered are listed in Table 1 and the meaning of the fault-mode abbreviations are given in Table 2

$\begin{array}{ll}B L & \text { Boost Leak } \\ M L & \text { Manifold Leak } \\ B B & \text { Boost Pressure Sensor Bias } \\ B A F & \text { Boost Pressure Sensor Arbitrary Fault } \\ M G & \text { Manifold Pressure Sensor Gain-Fault } \\ M C & \text { Manifold Pressure Sensor Cut-Off } \\ T L F & \text { Throttle Sensor Linear Fault } \\ A L C & \text { Air Mass-Flow Sensor Loose Contact }\end{array}$

Table 2: The component fault-mode abbreviations..

As was said before, only single (system) fault-modes are considered. The single fault assumption means that the set of system fault-modes is implicitly determined by Table 1 . The definitions of each system fault-mode, i.e. $\mathcal{M}_{\gamma}(\theta)$, are given in Section 5.1, where the construction of the hypothesis tests are described.
The fault state vector $\theta$ is

$$
\begin{aligned}
& \theta=\left[\theta_{B}, \theta_{M}, \theta_{B S}, \theta_{M S}, \theta_{T S}, \theta_{A S}\right]= \\
& {\left[k_{b}, k_{m},\left(b_{p_{b}}, c_{2}(t)\right), g_{p_{m}},\left(g_{\alpha}, b_{\alpha}\right), c_{1}(t)\right]}
\end{aligned}
$$

where $c_{1}(t)$ and $c_{2}(t)$ are signals while the other parameters are scalar constants. More information about the fault state vector is given in the following section.

\subsection{Description of the Hypothesis Tests}

From Section 3, we know that the problem of designing hypothesis test $H T_{\gamma}$ consists of determining $V_{\gamma}(\theta, \mathbf{x}), \Theta_{\gamma}$, and $J_{\gamma}$. Because of space limitations, only for some of the hypothesis tests, $V_{\gamma}(\theta, \mathbf{x})$ and $\Theta_{\gamma}$ are given below. Descriptions for the other hypothesis tests can be found in [6].

\section{No Fault NF}

The model $\mathcal{M}_{\mathbf{N F}}$ corresponding to this fault mode is obtained by using the fault-free model (7) in combination with

$$
\begin{aligned}
\dot{m}_{s} & =\dot{m} \\
p_{b, s} & =p_{b} \\
p_{m, s} & =p_{m} \\
\alpha_{s} & =\alpha \\
n_{s} & =n
\end{aligned}
$$

where the index $s$ denotes that for example $\dot{m}_{s}$ is the sensor signal in contrast to $\dot{m}$ which is the physical quantity. The fault mode NF corresponds to one specific value of $\theta$ which, in accordance with $(6)$, means that $T_{\mathbf{N F}}(\mathbf{x})=V_{\mathbf{N F}}(\mathbf{x})$. The measure $V_{\mathbf{N F}}(\mathbf{x})$ is defined as

$$
\begin{aligned}
V_{\mathbf{N F}}(\mathbf{x})=\frac{1}{N} & \sum_{t=1}^{N}\left(\dot{m}_{s}-f\left(p_{b, s}, \alpha_{s}, p_{m, s}\right)\right)^{2}+ \\
& +\frac{1}{N} \sum_{t=1}^{N}\left(f\left(p_{b, s}, \alpha_{s}, p_{m, s}\right)-g\left(p_{m, s}, n_{s}\right)\right)^{2}
\end{aligned}
$$

Note that, to simplify notation, we have dropped the timeargument of signals. The parameter space $\Theta_{\mathbf{N F}}$ is $\Theta_{\mathbf{N F}}=$ $\{[0,0,0, \mathbf{0}, 1,1,0, \mathbf{1}]\}$, where bold-face numbers denotes vectors.

Using this measure implies that if the present fault mode is $\mathbf{N F}$, then the test quantity becomes small and for all other fault modes, the test quantity becomes large. In a hypothesis test defined by (3) and (4) this means that $M_{\mathrm{NF}}=\{\mathbf{N F}\}$.

\section{Manifold Leak ML}

The model $\mathcal{M}_{\mathbf{M L}}\left(k_{m}\right)$ corresponding to this fault mode is given by (7a) and (8) together with the identities (9). The scalar parameter $k_{m}$ defines the area of the leakage and is constrained by $\left.\left.k_{m} \in \mathcal{D}_{M L}^{M}=\right] 0,0.5\right]$. The measure $V_{\mathbf{M L}}\left(k_{m}, \mathbf{x}\right)$ is

$$
\begin{aligned}
& V_{\mathbf{M L}}\left(k_{m}, \mathbf{x}\right)=\frac{1}{N} \sum_{t=1}^{N}\left(\dot{m}_{s}-f\left(p_{b, s}, \alpha_{s}, p_{m, s}\right)\right)^{2}+ \\
& +\frac{1}{N} \sum_{t=1}^{N}\left(f\left(p_{b, s}, \alpha_{s}, p_{m, s}\right)-g\left(p_{m, s}, n_{s}\right)+k_{m} h_{m}\left(p_{m, s}\right)\right)^{2}
\end{aligned}
$$


Using this measure means that $M_{\mathrm{ML}}=\{\mathbf{N F}, \mathbf{M L}\}$. The expression of $V_{\mathbf{M L}}\left(k_{m}, \mathbf{x}\right)$ contains two terms even though only one of them is affected by the fault. The reason to include the second term is, as was said in the end of Section 3.1, that we want the test quantity to become large for other faults, not belonging to fault mode $\mathbf{M L}$.

\section{Manifold Pressure Sensor Gain-Fault MG}

The model $\mathcal{M}_{\mathbf{M G}}\left(g_{p_{m}}\right)$ corresponding to this fault mode is obtained by using the fault free model (7) together with identities (9) but replacing (9c) with $p_{m, s}=g_{p_{m}} p_{m}$. The constraint on the scalar parameter $g_{p_{m}}$ is $g_{p_{m}} \in \mathcal{D}_{M G}^{M S}=$ $[0.5,1[\cup] 1,2]$. The measure $V_{\mathbf{M G}}\left(g_{p_{m}}, \mathbf{x}\right)$ is

$$
\begin{array}{r}
V_{\mathbf{M G}}\left(g_{p_{m}}, \mathbf{x}\right)=\frac{1}{N} \sum_{t=1}^{N}\left(\dot{m}_{s}-f\left(p_{b, s}, \alpha_{s}, p_{m, s} / g_{p_{m}}\right)\right)^{2}+ \\
\left.+\frac{1}{N} \sum_{t=1}^{N}\left(f\left(p_{b, s}, \alpha_{s}, p_{m, s} / g_{p_{m}}\right)-g\left(p_{m, s} / g_{p_{m}}, n_{s}\right)\right)\right)^{2}
\end{array}
$$

Using this measure means that $M_{\mathbf{M G}}=\{\mathbf{N F}, \mathbf{M G}\}$.

\section{Manifold Pressure Sensor Cut-Off MC}

This fault mode represents a cut-off in the electrical connection to the manifold pressure sensor. The model $\mathcal{M}_{M C}(\nu)$ corresponding to this fault mode is obtained by using the fault free model (7) together with identities (9) but replacing (9c) with $p_{m, s}=g_{p_{m}} p_{m}$. The parameter $g_{p_{m}}$ takes value 1 in the fault-free case and value 0 when there is a cut-off present. This means that $g_{p_{m}} \in \mathcal{D}_{M C}^{M S}=\{0\}$ when fault mode $\mathbf{M C}$ is present. This corresponds to exactly one value of $\theta$ which implies that $T_{\mathbf{M C}}(\mathbf{x})=V_{\mathbf{M C}}(\mathbf{x})$. The measure $V_{\mathbf{M C}}(\mathbf{x})$ is defined as

$$
V_{\mathbf{M C}}(\mathbf{x})=\frac{1}{N} \sum_{t=1}^{N} p_{b, s}^{2}
$$

Using this measure means that $M_{\mathbf{M C}}=\{\mathbf{M C}\}$.

\section{Air Mass-Flow Sensor Loose Contact ALC}

The model $\mathcal{M}_{\mathbf{A L C}}\left(c_{1}(t)\right)$ corresponding to this fault mode is obtained by using the fault free model $(7)$ together with identities (9) but replacing (9a) with $\dot{m}_{s}(t)=\dot{m}(t) c_{1}(t)$. The parameter $c_{1}(t)$ is a stochastic process taking values such that $c_{1}(t) \in\{0,1\}$. This means that the parameter space $\mathcal{D}_{A L C}^{A S}$ becomes $\mathcal{D}_{A L C}^{A S}=\{0,1\}^{N}-\{0\}^{N}$ and the measure $V_{\mathbf{A L C}}\left(c_{1}(t), \mathbf{x}\right)$ is

$$
\begin{aligned}
V_{\mathbf{A L C}}\left(c_{1}(t), \mathbf{x}\right) & =\frac{1}{N} \sum_{t=1}^{N}\left(\dot{m}_{s}-c_{1} f\left(p_{b, s}, \alpha_{s}, p_{m, s}\right)\right)^{2}+ \\
+ & \left.\frac{1}{N} \sum_{t=1}^{N}\left(f\left(p_{b, s}, \alpha_{s}, p_{m, s}\right)-g\left(p_{m, s}, n_{s}\right)\right)\right)^{2}
\end{aligned}
$$

Using this measure means that $M_{\mathbf{A L C}}=\{\mathbf{N F}, \mathbf{A L C}\}$

\section{Boost Pressure Sensor Arbitrary Fault BAF}

The model $\mathcal{M}_{\mathbf{B A F}}\left(c_{2}(t)\right)$ corresponding to this fault mode is obtained by using the fault free model $(7)$ together with identities (9) but replacing $(9 \mathrm{~b})$ with $p_{b, s}=p_{b}+c_{2}(t)$. The parameter $c_{2}(t)$ is a stochastic process taking arbitrary values. This means that the parameter space $\mathcal{D}_{B A F}^{B S}$ becomes $\mathcal{D}_{B A F}^{B S}=\{0\} \times\left(\mathbb{R}^{N}-\{0\}^{N}\right)$ and the measure $V_{\mathbf{B A F}}\left(c_{2}(t), \mathbf{x}\right)$ is

$$
\begin{gathered}
V_{\mathbf{B A F}}\left(c_{2}(t), \mathbf{x}\right)=\frac{1}{N} \sum_{t=1}^{N}\left(\dot{m}_{s}-f\left(p_{b, s}-c_{2}, \alpha_{s}, p_{m, s}\right)\right)^{2}+ \\
\left.+\frac{1}{N} \sum_{t=1}^{N}\left(f\left(p_{b, s}-c_{2}, \alpha_{s}, p_{m, s}\right)-g\left(p_{m, s}, n_{s}\right)\right)\right)^{2}
\end{gathered}
$$

Using this measure means that $M_{\mathbf{B A F}}=\{\mathbf{N F}, \mathbf{B B}, \mathbf{B A F}\}$.

\subsection{Practical Considerations}

The procedure to compute (5), i.e. to minimize $V_{\gamma}(\mathbf{x})$, has not been addressed so far. In many cases the minimization procedure required is quite straightforward. The technical details are not going to be discussed here, but the interested reader is referred to general optimization literature, e.g. [7], (see also the discussion in [3]). However, for some of the hypothesis tests defined above, the computational load of doing the actual minimization in (5) can be quite heavy. One solution is to use a two-step procedure presented below.

First find a $\hat{\theta}$ that minimizes another function $\bar{V}_{\gamma}(\theta, \mathbf{x})$, i.e.

$$
\hat{\theta}=\arg \min _{\theta \in \Theta_{\gamma}} \bar{V}_{\gamma}(\theta, \mathbf{x})
$$

Then use as test quantity $T_{\gamma}(\mathbf{x})=V_{\gamma}(\hat{\theta}, \mathbf{x})$.

If $\bar{V}_{\gamma}(\theta, \mathbf{x})$ is chosen so that the minimizing value $\hat{\theta}$, under $H_{\gamma}^{0}$, is close to the value that minimizes $V_{\gamma}(\theta, \mathbf{x})$, then it is reasonable to assume that

$$
\min _{\theta \in \Theta_{\gamma}} V_{\gamma}(\theta, \mathbf{x}) \approx V_{\gamma}(\hat{\theta}, \mathbf{x})
$$

This means that if we use the test quantity $T_{\gamma}(\mathbf{x})=V_{\gamma}(\hat{\theta}, \mathbf{x})$, we can expect approximately the same result compared to if (5) was used.

In the implementation of the hypothesis tests defined above, this two-step procedure is used in the tests $H T_{\mathbf{B L}}$, $H T_{\mathbf{M L}}, H T_{\mathbf{A L C}}$, and $H T_{\mathbf{B A F}}$. In all these four cases, $\bar{V}_{\gamma}(\theta, \mathbf{x})$ is chosen as one of the two terms in $V_{\gamma}(\theta, \mathbf{x})$.

For $H T_{\mathbf{M L}}, \bar{V}_{\mathbf{M L}}\left(k_{m}, \mathbf{x}\right)$ is

$$
\bar{V}_{\mathbf{M L}}\left(k_{m}, \mathbf{x}\right)=\frac{1}{N} \sum_{t=1}^{N}\left(\dot{m}_{s}-f\left(p_{b, s}, \alpha_{s}, p_{m, s}\right)\right)^{2}
$$

For the test $H T_{\mathbf{A L C}}, \bar{V}_{A L C}\left(c_{1}(t), \mathbf{x}\right)$ is

$$
V_{\mathbf{A L C}}\left(c_{1}(t), \mathbf{x}\right)=\frac{1}{N} \sum_{t=1}^{N}\left(\dot{m}_{s}-c_{1} f\left(p_{b, s}, \alpha_{s}, p_{m, s}\right)\right)^{2}
$$

This function can be conveniently minimized by choosing

$$
c_{1}= \begin{cases}0 & \dot{m}_{s}<\epsilon \\ 1 & \dot{m}_{s} \geq \epsilon\end{cases}
$$

For the test $H T_{\mathbf{B A F}}, \bar{V}_{B A F}\left(c_{2}(t), \mathbf{x}\right)$ is

$$
\bar{V}_{\mathbf{B A F}}\left(c_{2}(t), \mathbf{x}\right)=\frac{1}{N} \sum_{t=1}^{N}\left(\dot{m}_{s}-f\left(p_{b, s}-c_{2}, \alpha_{s}, p_{m, s}\right)\right)^{2}
$$


This function is conveniently minimized by choosing

$$
c_{2}(t)=f^{-1}\left(\dot{m}_{s}(t), \alpha_{s}(t), p_{m, s}(t)\right)-p_{b, s}
$$

where $f^{-1}\left(\dot{m}_{s}(t), \alpha_{s}(t), p_{m, s}(t)\right)$ is the "inverse" $f\left(p_{b, s}, \alpha_{s}, p_{m, s}\right)$ and gives an estimate of $p_{b, s}$.

\section{Experimental Validation}

The diagnosis system described in the previous section was implemented in Matlab and tested extensively on the automotive engine described in Section 4. Leaks were applied by using exchangeable bolts. One bolt were mounted in the wall of the manifold and the other in the wall of the air tube in front of the throttle. All other faults were emulated in software by applying appropriate changes to the sensor signals. For each fault mode, a number of different fault sizes were tested. Below we will describe off-line experiments. Also on-line experiments were performed and these are described in [6].

The data length was $N=1000$ which corresponds to 100 s. No special effort was made to find optimal threshold values $J_{\gamma}$; they were all chosen to be $J_{\gamma}=0.4$. With these choices, good functionality was obtained for all kinds of faults but because of space limitation, we have selected to present only one case: a manifold leak, i.e. system faultmode ML. The result of this case is shown in Table 3. Each row show the result of one individual hypothesis test $H T_{\gamma}$. The value of the test quantity $T_{\gamma}(\mathbf{x})$ for each hypothesis $H T_{\gamma}$ is shown in the second column. The threshold $J_{\gamma}$ is shown in the third column (in this example, all were chosen to the same value). If $T_{\gamma}(\mathbf{x})>J_{\gamma}$, i.e. $H_{\gamma}^{0}$ is rejected, then the fourth column shows the the set $M_{\gamma}^{C}$.

\begin{tabular}{llll}
$\gamma$ & $T_{\gamma}(\mathbf{x})$ & $J_{\gamma}$ & $M_{\gamma}^{C}$ \\
\hline NF & 0.4921 & 0.4 & ALC BAF BB BL MC MG ML TLF \\
BL & 0.4985 & 0.4 & ALC BAF BB MC MG ML TLF \\
ML & 0.1881 & 0.4 & \\
BB & 0.423 & 0.4 & ALC BAF BL MC MG ML TLF \\
MG & 0.328 & 0.4 & \\
MC & 3742 & 0.4 & ALC BAF BB BL MG ML NF TLF \\
TLF & 0.3623 & 0.4 & \\
ALC & 0.4921 & 0.4 & BAF BB BL MC MG ML TLF \\
BAF & 0.4642 & 0.4 & ALC BL MC MG ML TLF \\
\hline
\end{tabular}

Diagnosis Statement: MG ML TLF

Table 3: The hypothesis tests and the diagnosis statement for fault mode ML.

For the case shown in the table, the hypotheses $H_{\mathbf{N F}}^{0}$, $H_{\mathbf{B L}}^{0}, H_{\mathbf{B B}}^{0}, H_{\mathbf{M C}}^{0}, H_{\mathbf{A L C}}^{0}$, and $H_{\mathbf{B A F}}^{0}$ are rejected. Applying the intersection of the decision logic, i.e. (2), implies that the diagnosis statement contains 3 possible system fault-modes that can explain the behavior of the process.

The actual fault was fairly small, which is reflected in the result that it could not be uniquely isolated. The diagnosis statement contains the fault modes MG, ML, and TLF. This should be interpreted as that in addition to the present fault mode $\mathbf{M L}$, the fault modes MG and TLF can also explain the behavior of the process. In other experiments, where the fault was larger or the system was excited more, the fault mode $\mathbf{M L}$ could be uniquely isolated.

\section{Conclusions}

A framework and a method for describing and solving diagnosis problems has been suggested. The framework formalizes the description of faults and then the diagnosis problem is solved by the method which is based on hypothesis testing.

Previous methods are each specialized for one type of faults. In contrast, the principles presented here, are capable of handling a large variety of different faults. Therefore they are also more useful in real applications, where typically many different types of faults need to be considered. In addition, more model knowledge can be included in the design of the diagnosis system, which implies that there is a large potential to obtain better diagnosis performance. Both the framework and the method are also quite general and several other principles of diagnosis can be seen as special cases, e.g. parameter estimation, structured residuals [1], and statistical methods.

The framework and the method are applied to the design of a diagnosis system for the air-intake system of an automotive engine. This application clearly shows the strength of these principles because several different types of sensor faults and leakage need to be diagnosed. With previous methods presented in [4] and [5], this was not possible. The features of the constructed diagnosis system are demonstrated by using experiments on a real automotive engine.

\section{Acknowledgments}

This research is supported by NUTEK (Swedish National Board for Industrial and Technical Development) and SAAB Automobile AB.

\section{References}

[1] J. Gertler. Analytical redundancy methods in fault detection and isolation; survey and synthesis. IFAC Fault Detection, Supervision and Safety for Technical Processes, pages 9-21, Baden-Baden, Germany, 1991.

[2] R.J. Patton. Robust model-based fault diagnosis:the state of the art. IFAC Fault Detection, Supervision and Safety for Technical Processes, pages 1-24, Espoo, Finland, 1994.

[3] M. Nyberg. Model Based Fault Diagnosis: Methods, Theory, and Automotive Engine Applications. PhD thesis, Department of Electrical Engineering, Linköping University, Linköping, Sweden, 1999.

[4] M. Nyberg and L. Nielsen. Model based diagnosis for the air intake system of the SI-engine. SAE Paper, (970209), 1997.

[5] M. Nyberg and A. Perkovic. Model based diagnosis of leaks in the air-intake system of an SI-engine. $S A E P a$ per, (980514), 1998.

[6] M. Nyberg. Model based diagnosis of both sensor-faults and leakage in the air-intake system of an SI-engine. SAE Paper, (1999-01-0860), 1999.

[7] D. Luenberger. Linear and Nonlinear Programming. Addison Wesley, 1989. 